\title{
The burden of severe asthma in childhood and adolescence: results from the paediatric U-BIOPRED cohorts
}

\author{
Louise Fleming ${ }^{1,2,3}$, Clare Murray ${ }^{4}$, Aruna T. Bansal ${ }^{5}$, Simone Hashimoto ${ }^{6}$, Hans Bisgaard ${ }^{7,8}$, Andrew Bush ${ }^{1,3,9,10}$ \\ Urs Frey ${ }^{11}$, Gunilla Hedlin ${ }^{12}$, Florian Singer ${ }^{13,14}$, Wim M. van Aalderen ${ }^{15}$, Nadja H. Vissing ${ }^{7,8}$, \\ Zaraquiza Zolkipli ${ }^{16,17,18}$, Anna Selby ${ }^{16,17,18}$, Stephen Fowler ${ }^{4,19}$. Dominick Shaw ${ }^{20}$, Kian Fan Chung ${ }^{1,2,3}$, \\ Ana R. Sousa ${ }^{21}$, Scott Wagers ${ }^{22}$, Julie Corfield ${ }^{23,24}$, Ioannis Pandis ${ }^{25}$. Anthony Rowe ${ }^{26}$, Elena Formaggio ${ }^{27}$, \\ Peter J. Sterk ${ }^{6}$ and Graham Roberts ${ }^{16,17,18}$ on behalf of the U-BIOPRED Study Group ${ }^{28}$
}

\begin{abstract}
Affiliations: ${ }^{1}$ National Heart and Lung Institute, Imperial College London, London, UK. ${ }^{2}$ NIHR Biomedical Research Unit, Royal Brompton and Harefield NHS Trust, London, UK. ${ }^{3}$ NIHR Biomedical Research Unit, Royal Brompton NHS Trust, London, UK. ${ }^{4}$ Centre for Respiratory Medicine and Allergy, The University of Manchester, Manchester Academic Health Science Centre, University Hospital of South Manchester NHS Foundation Trust, Manchester, UK. ${ }^{5}$ Acclarogen Ltd, St John's Innovation Centre, Cambridge, UK. ${ }^{6}$ Dept of Respiratory Medicine, Academic Medical Centre, University of Amsterdam, Amsterdam, The Netherlands. ${ }^{7}$ Copenhagen Prospective Studies on Asthma in Childhood, Faculty of Health and Medical Sciences, University of Copenhagen, Copenhagen, Denmark. ${ }^{8}$ Gentofte Hospital, University of Copenhagen, Copenhagen, Denmark. ${ }^{9}$ Dept of Paediatrics, Imperial College London, London, UK. ${ }^{10}$ Dept of Respiratory Paediatrics, Royal Brompton Hospital, London, UK. "1'University Children's Hospital Basel UKBB, University of Basel, Basel, Switzerland. ${ }^{12}$ Dept of Women's and Children's Health and Center for Allergy Research, Karolinska Institutet at Karolinska University Hospital, Stockholm, Sweden. ${ }^{13}$ University Children's Hospital Zurich, Zurich, Switzerland. ${ }^{14}$ University Children's Hospital Bern, Bern, Switzerland. ${ }^{15}$ Dept of Paediatric Respiratory Medicine and Allergy, Emma Children's Hospital, Academic Medical Centre, University of Amsterdam, Amsterdam, The Netherlands. ${ }^{16} \mathrm{NIHR}$ Southampton Respiratory Biomedical Research Unit, Clinical and Experimental Sciences and Human Development and Health, Southampton, UK. ${ }^{17}$ Faculty of Medicine, University of Southampton, Southampton, UK. ${ }^{18}$ The David Hide Asthma and Allergy Research Centre, St Mary's Hospital, Isle of Wight, UK. ${ }^{19}$ Airways Clinic, Lancashire Teaching Hospitals NHS Foundation Trust, Preston, UK. ${ }^{20}$ Respiratory Research Unit, University of Nottingham, Nottingham, UK. ${ }^{21}$ Respiratory Therapeutic Unit, GlaxoSmithKline, Stockley Park, UK. ${ }^{22}$ BioSci Consulting, Maasmechelen, Belgium. ${ }^{23}$ AstraZeneca R\&D, Mölndal, Sweden. ${ }^{24}$ Areteva, Nottingham, UK. ${ }^{25}$ Data Science Institute, South Kensington Campus, Imperial College London, London, UK. ${ }^{26}$ Janssen R\&D Ltd, High Wycombe, UK. ${ }^{27}$ CROMSOURCE, Verona, Italy. ${ }^{28}$ For a full list of members of the U-BIOPRED Study Group, please refer to the Acknowledgements section.
\end{abstract}

Correspondence: Graham Roberts, Paediatric Allergy and Respiratory Medicine (Mailpoint 805), Southampton University Hospital NHS Foundation Trust, Tremona Road, Southampton, S016 6YD, UK.

E-mail: g.c.roberts@soton.ac.uk

@ERSpublications

Children with severe preschool wheeze or severe asthma are usually atopic and have impaired quality of life http://ow.ly/RrrGE

This article has been amended according to the correction published in the June 2017 issue of the European Respiratory Journal.

This article has supplementary material available from erj.ersjournals.com

Received: May 182015 | Accepted after revision: Aug 232015 | First published online: Sept 242015

This study is registered on ClinicalTrials.gov (NCT01982162).

Support statement: The research leading to these results has received support from the Innovative Medicines Initiative (IMI) Joint Undertaking, under grant agreement no. 115010, resources for which are composed of financial contribution from the European Union's Seventh Framework Programme (FP7/2007-2013) and kind contributions from companies in the European Federation of Pharmaceutical Industries and Associations (EFPIA) (www.imi.europa.eu). A. Bush [CP5] was supported by the NIHR Respiratory Disease Biomedical Research Unit at the Royal Brompton and Harefield NHS Foundation Trust and Imperial College London. Help in data and knowledge management was received from the IMI-funded eTRIKS project (EU grant code no. 115446). In Southampton, support was received from the University Hospital NHS Foundation Trust and the NIHR-Wellcome Trust Clinical Research Facility.

Conflict of interest: Disclosures can be found alongside the online version of this article at erj.ersjournals.com

Copyright OERS 2015 
ABSTRACT U-BIOPRED aims to characterise paediatric and adult severe asthma using conventional and innovative systems biology approaches.

A total of 99 school-age children with severe asthma and 81 preschoolers with severe wheeze were compared with 49 school-age children with mild/moderate asthma and 53 preschoolers with mild/ moderate wheeze in a cross-sectional study.

Despite high-dose treatment, the severe cohorts had more severe exacerbations compared with the mild/ moderate ones (annual medians: school-aged 3.0 versus 1.1, preschool 3.9 versus $1.8 ; \mathrm{p}<0.001$ ). Exhaled tobacco exposure was common in the severe wheeze cohort. Almost all participants in each cohort were atopic and had a normal body mass index. Asthma-related quality of life, as assessed by the Paediatric Asthma Quality of Life Questionnaire (PAQLQ) and the Paediatric Asthma Caregiver's Quality of Life Questionnaire (PACQLQ), was worse in the severe cohorts (mean \pm SE school-age PAQLQ: $4.77 \pm 0.15$ versus $5.80 \pm 0.19$; preschool PACQLQ: $4.27 \pm 0.18$ versus $6.04 \pm 0.18$; both $\mathrm{p} \leqslant 0.001$ ); however, mild/moderate cohorts also had significant morbidity. Impaired quality of life was associated with poor control and airway obstruction. Otherwise, the severe and mild/moderate cohorts were clinically very similar.

Children with severe preschool wheeze or severe asthma are usually atopic and have impaired quality of life that is associated with poor control and airflow limitation: a very different phenotype from adult severe asthma. In-depth phenotyping of these children, integrating clinical data with high-dimensional biomarkers, may help to improve and tailor their clinical management.

\section{Introduction}

Asthma is one of the most common chronic diseases in childhood. Although many achieve control with currently available therapies, an estimated $5-10 \%$ of patients remain symptomatic despite receiving large amounts of treatment. These children with severe asthma [1] have poor quality of life (QoL), frequent asthma attacks and lung function impairment, are at high risk of side-effects from medication and account for significant medical and societal costs.

It is increasingly recognised that asthma, and particularly severe asthma, is not one single disease entity. Data in adults have been available for some time [2] but evidence now exists in children to suggest that there are a number of different clinical manifestations of severe asthma that are driven by a variety of pathophysiological mechanisms [3, 4]. Phenotypic classifications in children have primarily been based on clinical data, lung function measurement and assessment of allergic status. The small number of studies that have included biological samples have described important differences in the underlying pathobiology of severe asthma in children compared with adults [5-7]. Some but not all preschool children with severe wheeze have evidence of airway remodelling and inflammation from an early age [8], consistent with established asthma, but little is known about the underlying mechanisms, which in many cases appear to be very different from school-age and adult asthma. These early changes do not always predict a progression to asthma [9]. These observations are indicative of considerable heterogeneity amongst children with severe school-aged asthma or severe preschool wheeze.

In order to capture the relevant phenotypes of children with severe asthma or severe wheeze, careful and extensive clinical characterisation is required. This provides the basis for future integration with biological disease markers. The Unbiased Biomarkers for the Prediction of Respiratory Disease Outcomes (U-BIOPRED) project is a public-private partnership, within the framework of the Innovative Medicines Initiative, bringing together academic institutions and European Federation of Pharmaceutical Industries and Associations partners from across Europe. It was set up in 2009 in order to take advantage of the combination of extensive clinical characterisation and biological fingerprinting by "omics" technologies for the unbiased discovery of phenotypes in both adult and paediatric severe asthma [10]. The paediatric arm of the U-BIOPRED study used the same thorough clinical characterisation and innovative systems biology approach as the adult study [11] to integrate clinical, physiological and inflammatory data and patient/ parent-reported outcomes with the high-dimensional data of "omics" technologies (transcriptomic, proteomic, lipidomic and metabolomic) obtained from blood, urine, breath and airway samples [12].

The main objective of this first report of the paediatric U-BIOPRED study was to fully clinically characterise the cohorts of children with severe asthma and preschool wheeze and mild/moderate cohorts based on cross-sectional baseline data. The second objective was to investigate the burden of severe asthma and severe preschool wheeze, as described by QoL, and the clinical factors that relate to this burden.

\section{Methods}

This was a multicentre, prospective, observational cohort study following the life course of asthma. Full details of the adult cohorts are described in the companion paper by SHAW et al. [11]. 


\section{Cohorts}

Seven centres in five European countries recruited preschool (age 1-5 years) and school-age (age 617 years) children. Four paediatric cohorts were recruited by approaching consecutive patients attending respiratory and general paediatric clinics who fulfilled the following inclusion criteria. 1) Severe school-aged asthma (SA): ongoing poorly controlled asthma (persistent symptoms, frequent exacerbations or persistent airflow limitation) despite high-dose inhaled corticosteroids (ICS) and at least two other controller medications [13]. 2) Mild/moderate school-aged asthma (MMA): controlled or partly controlled asthma, prescribed low-dose ICS and no other or one additional controller medication. 3) Severe preschool wheeze (SW): persistent symptoms and frequent exacerbations despite current or failed high-dose ICS and a leukotriene receptor antagonist (LTRA). 4) Mild/moderate preschool wheeze (MMW): controlled or partially controlled symptoms prescribed no treatment or low-dose ICS and/or a LTRA.

Full cohort descriptions, inclusion and exclusion criteria are shown in table S2 in the online supplementary material. All children in the severe cohorts (SA and SW) had been under follow-up with a respiratory paediatrician for $\geqslant 6$ months before enrolling in the study. During this time, assessments were undertaken to exclude other diagnoses, treat comorbidities, optimise asthma control, assess medication adherence (e.g. checking prescription uptake) and reduce allergen exposure in sensitised individuals [13].

\section{Study design}

The study was approved by the local ethics committees (see table S1). Parents/caregivers provided written consent; children gave assent where appropriate. The study is registered with ClinicalTrials.gov (NCT01982162). The study aims and outcomes have been published on the U-BIOPRED website (www.europeanlung.org/en/projects-and-research/projects/u-biopred/home).

All participants were identified and recruited locally and attended a screening and baseline visit. Clinical data and biological samples were collected from all cohorts (figure 1). Full details are provided in the supplementary material.

\section{Study assessments}

Baseline data included demographics, current and past medical history (including detailed asthma and atopic disease history), medications, birth history, family history, exposure to environmental tobacco smoke, and known clinical and environmental risk factors. Asthma control was assessed using the Asthma Control Test (ACT) (for children $\geqslant 12$ years of age) [14] or the Childhood Asthma Control Test (cACT) for children <12 years [15]. Non-scheduled healthcare utilisation was assessed by documenting exacerbations. QoL was assessed using the Paediatric Asthma Quality of Life Questionnaire (PAQLQ) (school-aged children only) and the Paediatric Asthma Caregiver's Quality of Life Questionnaire

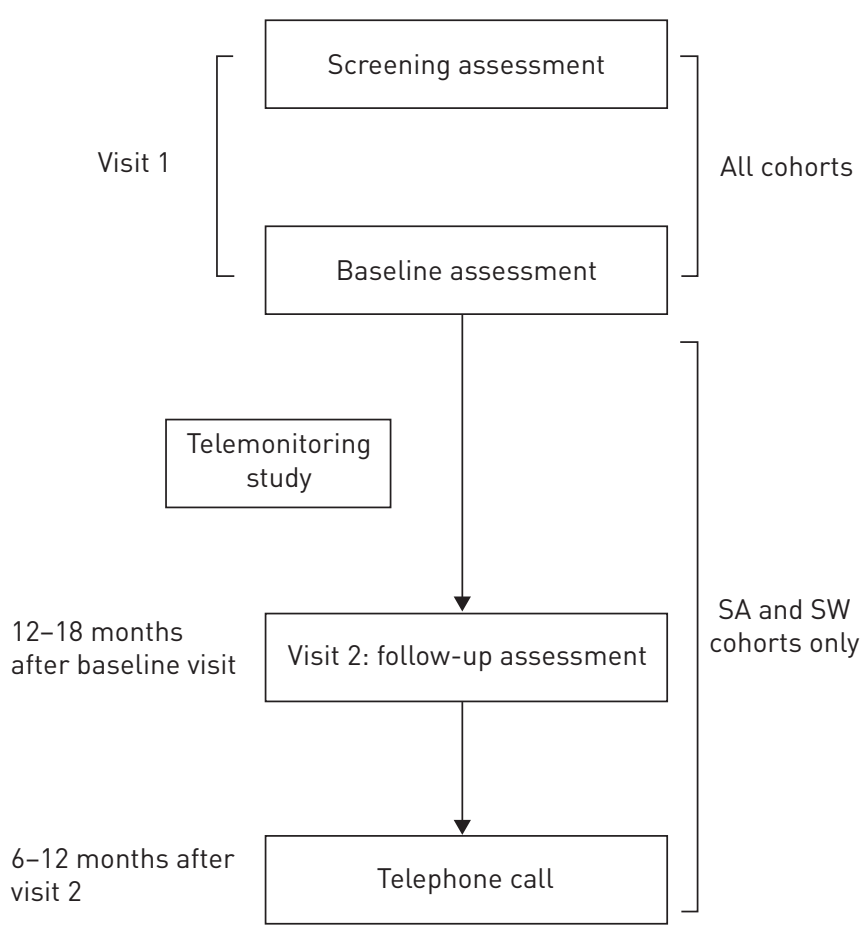

FIGURE 1 Visit schedule. SA: severe school-aged asthma; SW: severe preschool wheeze. 
(PACQLQ) [16, 17]. Adherence was evaluated using the Medication Adherence Report Scale (MARS) [18]. A summary of the assessments carried out in each of the cohorts is shown in table S3.

In all cohorts, total IgE, specific IgE tests and/or skin prick testing (SPT) to six common allergens were undertaken. Atopy was defined as the presence of sensitisation on SPT (wheal $\geqslant 3 \mathrm{~mm}$ ) or serum specific $\operatorname{IgE}$ $\left(\geqslant 0.35 \mathrm{kU} \cdot \mathrm{L}^{-1}\right)$. Spirometry before and after bronchodilator [19] and exhaled nitric oxide fraction (FeNO) were measured where possible. Sputum induction was performed in the school-aged cohorts and differential cell counts were determined. Exposure to environmental tobacco smoke was assessed by measuring urinary cotinine. In selected centres, forced oscillation technique and plethysmography were undertaken.

Full details of the methods are provided in the supplementary material, including samples collected for future "omic" analysis. A centralised biobank was selected for the study and operated in accordance with its own Standard Operating Procedures, as described in the supplementary material.

\section{Data management and statistics}

Data were entered into an electronic clinical record form before being transferred to the tranSMART system for quality control checks [20]. Missing data were not imputed.

The cohort sizes of 97 and 43 (comparing SA and MMA), and 77 and 54 (comparing SW and MMW), both provide $80 \%$ power to detect a difference in means of half a standard deviation, assuming standard normally distributed data, in a two-sided t-test at the 5\% significance level [21].

Due to the descriptive (as opposed to inferential) nature of the analyses presented, raw, unadjusted p-values are reported throughout the manuscript. Those in tables 1-4 were derived using logistic regression (binary variables) or general linear regression (continuous variables). Continuous variables exhibiting positive skew were summarised by the median and interquartile range (IQR), and were log-transformed prior to association testing. Where appropriate, tests of association were performed both with and without adjustments for age and sex.

Associations between key potential facets of asthma (forced expiratory volume in $1 \mathrm{~s}$ (FEV1) z-score, FEV1/forced vital capacity (FVC), age of onset/diagnosis, number of exacerbations in preceding 12 months, ACT z-score, body mass index (BMI), MARS, hay fever, eczema, atopy, smoking and white race) were each assessed singly for association with asthma burden, as quantified by QoL, using linear regression. Adjustments for age and sex were not applied at this stage due to a lack of univariate association between either age or sex. QoL contour plots were derived for continuous variables with $\mathrm{p}<0.05$, using two-dimensional kernel density estimation with a bivariate normal kernel, evaluated at 50 grid points in each direction [23]. The variables were also modelled jointly in a multivariate general linear model. Backwards stepwise regression using the Akaike Information Criterion was then applied, in order to derive a parsimonious model.

Analyses were performed using R version 2.15.2 ( $\mathrm{R}$ Core Team; www.r-project.org). The present report is based on cross-sectional analysis of the baseline data.

\section{Results}

\section{Participants}

A total of 298 children and teenagers with asthma or wheeze were screened to recruit 282 participants. Numbers of participants in each cohort that provided baseline questionnaire data, spirometry, blood samples and sputum samples are detailed in figure 2.

Cohorts SA and MMA were well matched for age (mean 12.21 and 11.26 years, respectively), as were cohorts SW and MMW (mean 3.56 and 3.46 years, respectively). Exposure to environmental tobacco smoke was reported by $15.8-22.8 \%$ of each cohort. More of the SW cohort were positive for urinary cotinine than of the MMW cohort (19.4\% versus 4.3\%; $\mathrm{p}=0.035)$ (table 1$)$.

\section{Atopy}

Most of the school-age participants in both cohorts (SA, MMA) were atopic (85.4\% and $89.5 \%$, respectively) (table 1). Rates of atopy were lower in both preschool cohorts (36.5\% and 37.5\%) (table 1 ). The majority of the school-age children (SA, MMA) had a diagnosis of eczema, hay fever or allergic rhinitis (table 1). Most of the preschoolers had a co-existing diagnosis of eczema with a third also having allergic rhinitis. In the preschool children, significantly more SW than MMW participants had a diagnosis of hay fever $(58.8 \%$ versus $36.1 \%$, respectively; $\mathrm{p}=0.04)$. A sizeable minority of participants reported symptoms of food allergy (40.2\% for SA versus $32.6 \%$ for MMA, $\mathrm{p}=0.39 ; 21.1 \%$ for SW versus $27.8 \%$ for MMW, $\mathrm{p}=0.38$ ). 
TABLE 1 Baseline demographic characteristics and medical history

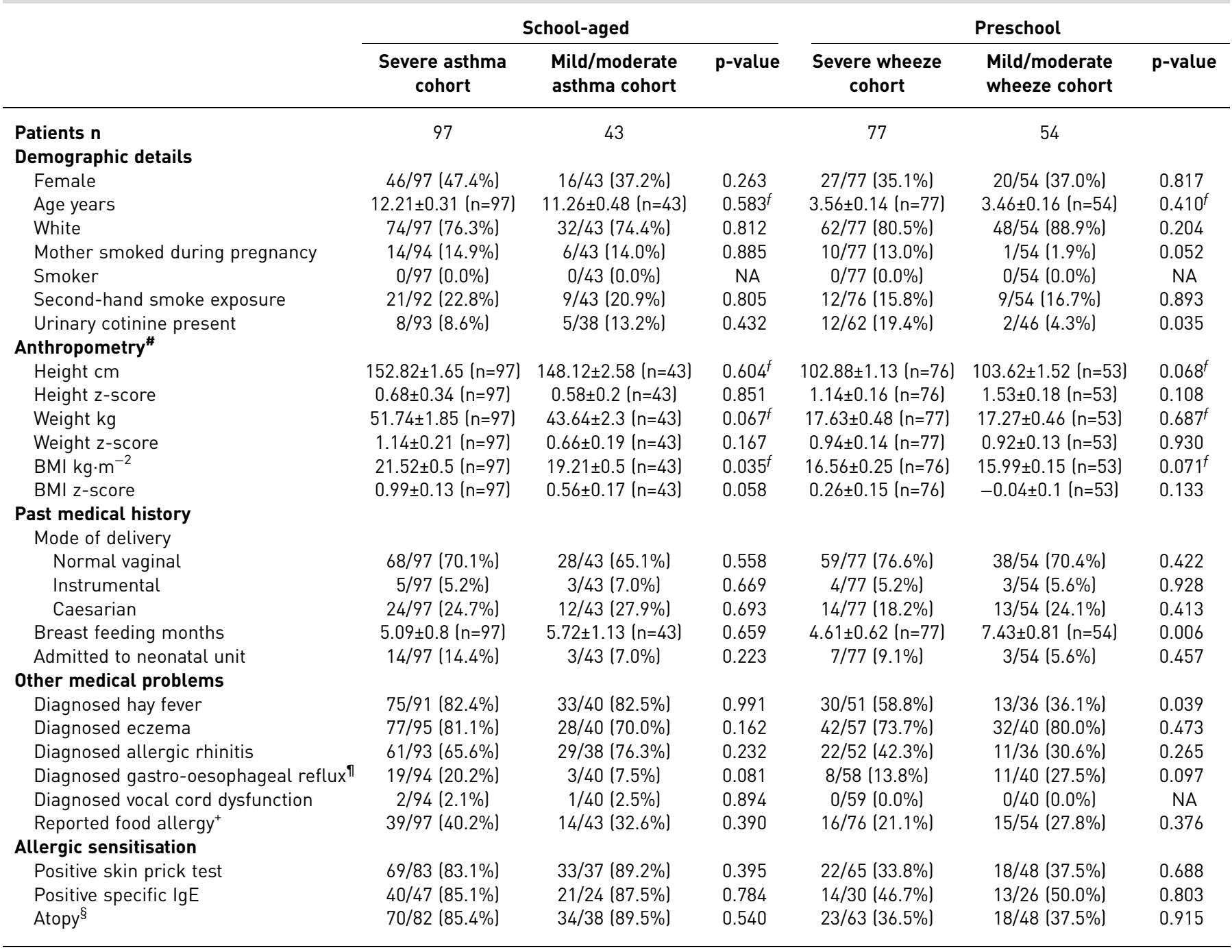

Data are presented as $\mathrm{n} / \mathrm{N}(\%)$ or mean $\pm \mathrm{SE}$, unless otherwise stated. p-values were calculated using Pearson's Chi-squared test or a Kruskall-Wallis test. BMI: body mass index; NA: not applicable. \#: anthropometry z-scores generated using the 1990 British growth data [22]; П: gastro-oesophageal reflux was diagnosed on the basis of suggestive symptoms, $\mathrm{pH}$ monitoring, endoscopy or response to therapy; ${ }^{+}$: symptoms of reported food allergy represent symptoms of urticaria, angioedema, pruiritis, throat tightness, stridor, chest tightness or wheeze within $2 \mathrm{~h}$ of contact with food; ${ }^{\S}$ : atopy defined as a positive skin prick test $(\geqslant 3 \mathrm{~mm})$ or a positive specific $\lg \mathrm{E}\left(\geqslant 0.35 \mathrm{kU} \cdot \mathrm{L}^{-1}\right)_{;}{ }^{f}$ : $\mathrm{p}$-values adjusted for age and sex.

\section{Asthma history and treatment}

The mean age at diagnosis was in the fourth year of life for both school-aged cohorts, whereas for the preschool ones it was in their second year (table 2). There were significant differences in the triggers for respiratory symptoms between the severe and mild/moderate cohorts (table 2). While almost all of cohorts SA, MMA and SW were treated with ICS, they were prescribed for less than half of MMW as most had failed to respond to ICS therapy. Additionally, $23.7 \%$ of SA and $5.2 \%$ of SW were receiving maintenance oral corticosteroid therapy. Parent/participant-reported adherence to therapy was good in all cohorts (table 2).

\section{Lung function and airway inflammation}

Lung function and bronchodilator reversibility in the SA and MMA cohorts were similar at baseline when participants were well (table 3). For preschool participants able to perform spirometry, results were again similar for severe and mild/moderate cohorts. There was a trend towards specific airway conductance being lower in the SA cohort compared with the MMA cohort (1.58 versus $\left.1.95 \mathrm{kPa} \cdot \mathrm{s}^{-1} ; \mathrm{p}=0.054\right)$ (table 3). We were only able to collect induced sputum from a minority of school-aged participants so we could not make a meaningful comparison between the cohorts (table 3 ). 


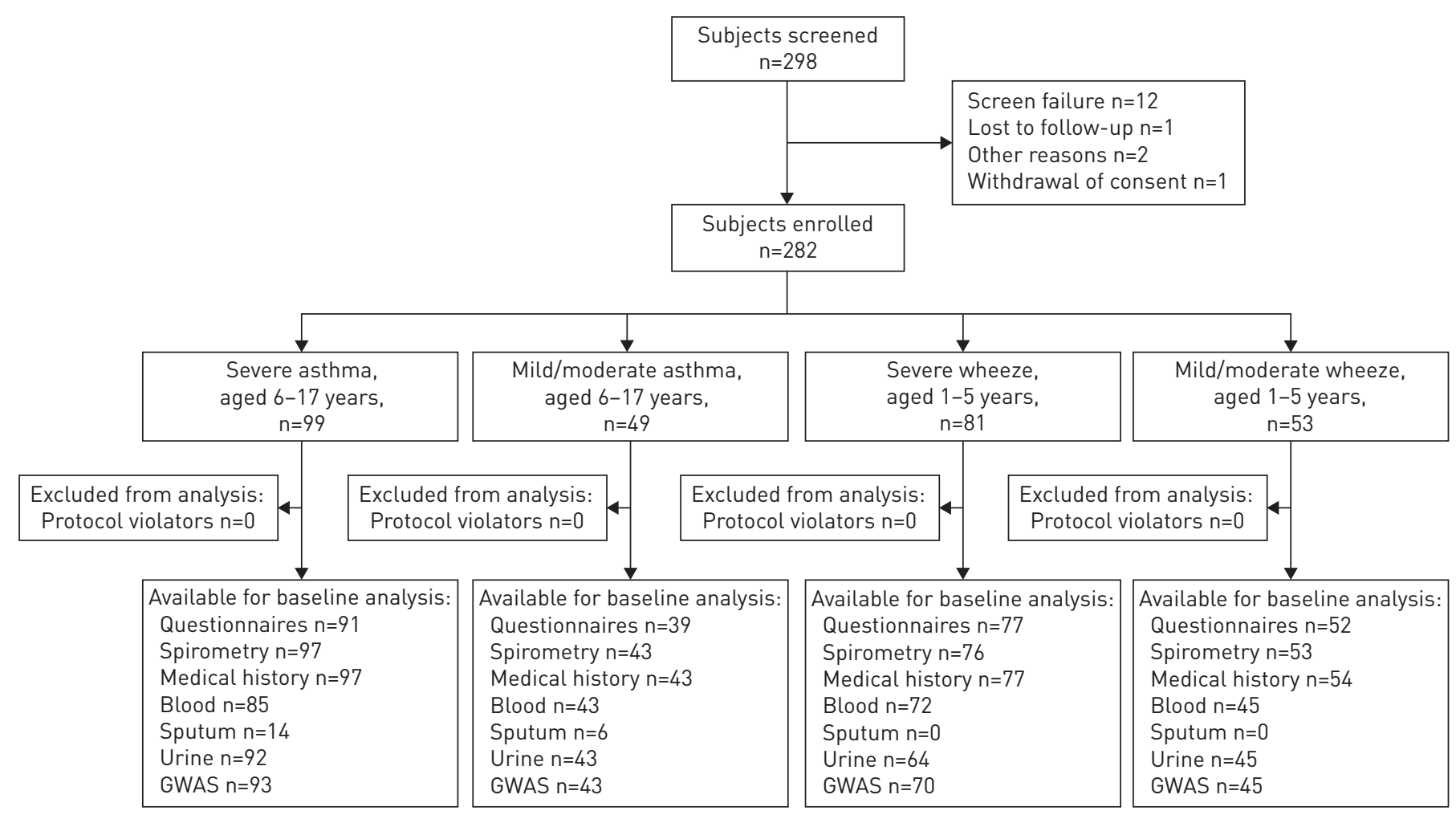

FIGURE 2 Consort diagram for participants in the paediatric Unbiased Biomarkers for the Prediction of Respiratory Disease Outcomes (U-BIOPRED) study. GWAS: genome-wide association study.

Asthma burden: QoL, control and exacerbations

Asthma-related QoL was used as the primary measure of burden. The mean result for the PAQLQ for the SA cohort was 4.77, equivalent to "somewhat bothered", significantly worse than for the MMA cohort (5.8, equivalent to "bothered a bit"; $\mathrm{p}<0.001)$. Similar differences were found for the symptoms, emotions and activity domains (table 4). For the preschool cohorts the PACQLQ was used as a proxy, given that there is no validated QoL tool for preschool wheeze. For SW the mean was 4.27 ("some of the time" or "somewhat worried/concerned"), again significantly worse than for MMW (6.04, "hardly ever" or "hardly worried/concerned"; $\mathrm{p}<0.001)$.

The burden of asthma was also illustrated by the ACT results, which assessed ongoing symptoms and rescue medication. Most of the severe cohorts were uncontrolled (74.6\% in SA compared with $29.2 \%$ in MMA, $\mathrm{p}<0.001 ; 78.0 \%$ in SW compared with $18.2 \%$ in MMW, $\mathrm{p}<0.001$ ). This was reflected in the number of exacerbations in the year prior to assessment. In the previous year, the SA cohort had a median of three exacerbations (IQR two to five), compared with one (IQR zero to two) in the MMA cohort $(\mathrm{p}<0.001)$. A similar difference was seen between the SW and MMW cohorts (table 4). However, there was still an important asthma burden in the mild/moderate cohorts.

Which factors are associated with asthma burden?

Asthma burden is described as asthma-related QoL, with z-scores used to give a combined variable for all age groups. Pre-bronchodilator FEV1, but not FEV1/FVC ratio, was significantly related to QoL (regression coefficient $0.151, \mathrm{p}=0.002$ ) (table 5). The number of exacerbations in the previous year was significantly inversely associated with asthma QoL $(-0.52, \mathrm{p}<0.001)$. Asthma control (measured by ACT and cACT $\mathrm{z}$-score) was significantly related to asthma QoL $(0.730, \mathrm{p}<0.001)$. BMI was inversely associated with asthma QoL $(-0.036, \mathrm{p}=0.011)$. These are illustrated in figure 3. Results were similar when PAQLQ and PACQLQ were considered separately (table S4).

To assess which factors were independent predictors of asthma-related QoL, a backward stepwise regression analysis was performed for FEV1 z-score, FEV1/FVC, age of onset/diagnosis, number of exacerbations in preceding 12 months, ACT z-score, BMI, MARS, hay fever, eczema, atopy, smoking and white race. Significant factors in the reduced model were ACT z-score (regression coefficient 0.76, $\mathrm{p}<0.001)$ and FEV1 z-score $(0.11, \mathrm{p}=0.036)$. 
TABLE 2 Asthma history and treatment

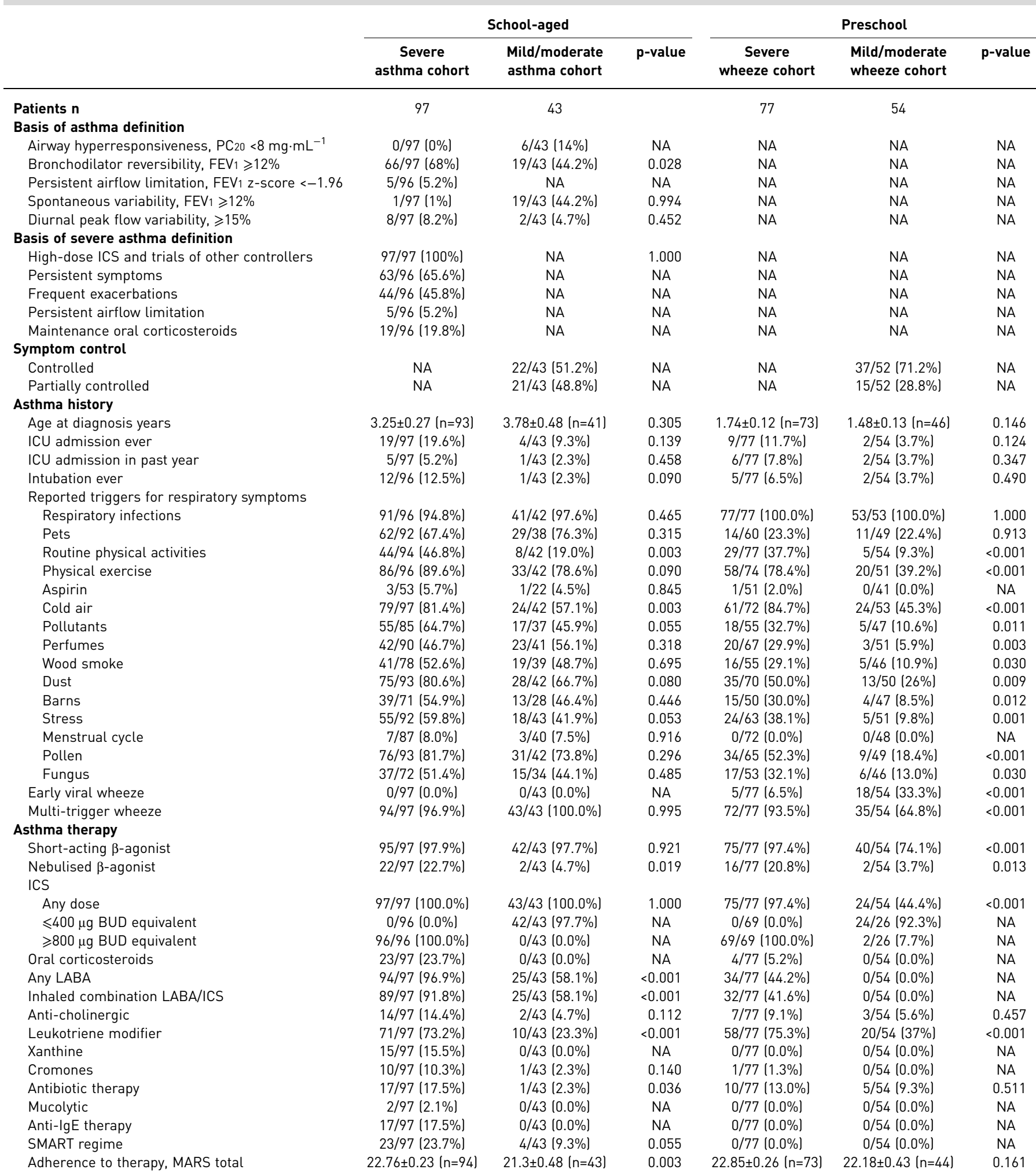

Data are presented as $\mathrm{n} / \mathrm{N}(\%)$ or mean $\pm \mathrm{SE}$, unless otherwise stated. $\mathrm{p}$-values were calculated using a two-sample t-test or a Chi-squared test. PC20: provocative concentration causing a $20 \%$ fall in FEV1; FEV1: forced expiratory volume in $1 \mathrm{~s}$; ICS: inhaled corticosteroids; ICU: intensive care unit; BUD: budesonide; LABA: long-acting $\beta$-agonist; SMART: Symbicort Maintenance and Reliever Therapy (AstraZeneca); MARS: Medication Adherence Report Scale la five-item self-report scale for assessment of adherent behaviour including unintentional and intentional non-adherence; each item was answered using a five-graded response scale (very often (1) to never (5)), so low scores indicate low levels of adherence); NA: not applicable. 
TABLE 3 Lung function and airway inflammation

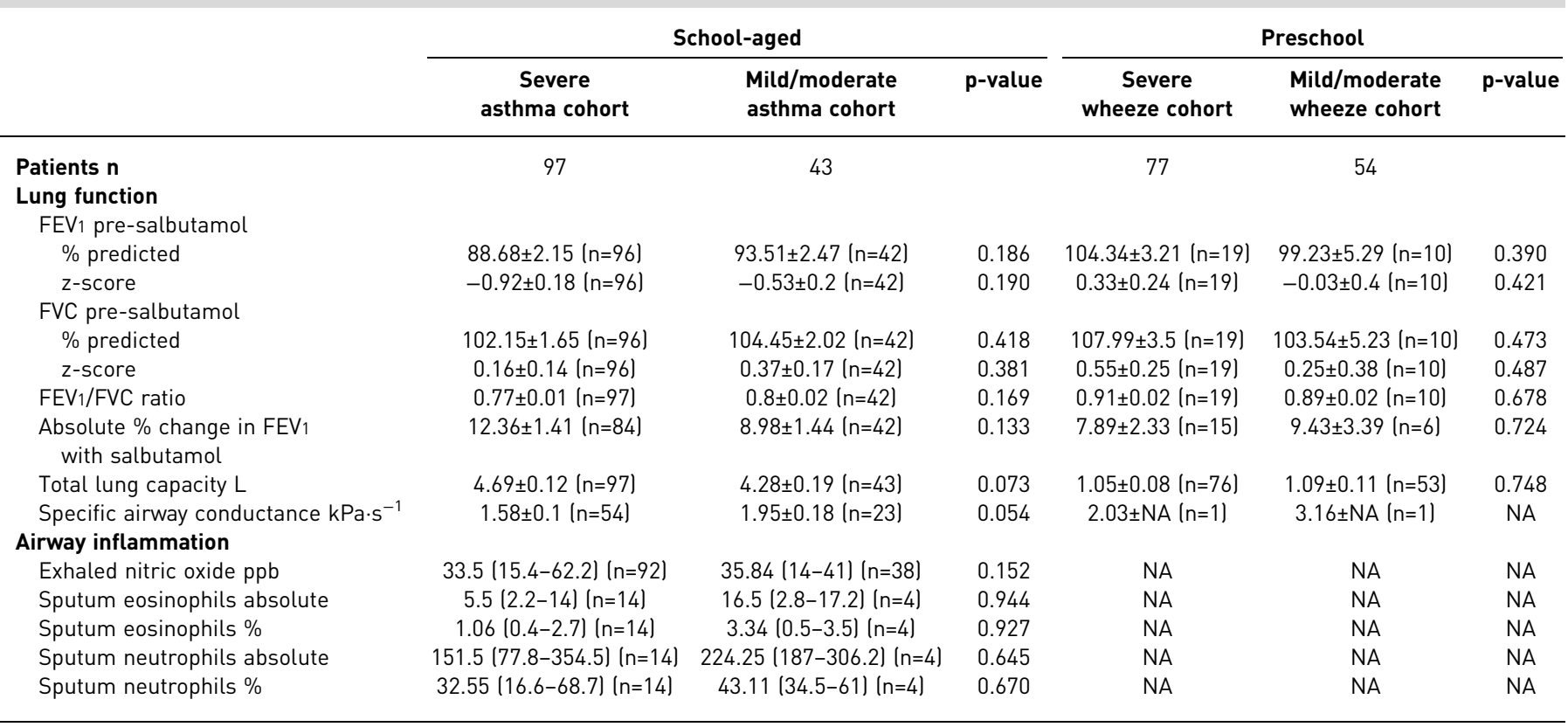

Data are presented as mean \pm SE or median (interquartile range), unless otherwise stated. Predictive lung function equations from QuANJER et al. [19] were used to generate predicted values and z-scores. p-values were calculated using a Kruskall-Wallis test. FEV1: forced expiratory volume in $1 \mathrm{~s}$; FVC: forced vital capacity; NA: not applicable.

TABLE 4 Asthma quality of life, exacerbations and control

\begin{tabular}{|c|c|c|c|c|c|c|}
\hline & \multicolumn{3}{|c|}{ School-aged } & \multicolumn{3}{|c|}{ Preschool } \\
\hline & $\begin{array}{c}\text { Severe } \\
\text { asthma cohort }\end{array}$ & $\begin{array}{l}\text { Mild/moderate } \\
\text { asthma cohort }\end{array}$ & p-value & $\begin{array}{c}\text { Severe } \\
\text { wheeze cohort }\end{array}$ & $\begin{array}{l}\text { Mild/moderate } \\
\text { wheeze cohort }\end{array}$ & p-value \\
\hline \multicolumn{7}{|l|}{$\begin{array}{l}\text { Asthma-related quality of life } \\
\text { PAQLQ }\end{array}$} \\
\hline Total mean & $4.77 \pm 0.15(n=91)$ & $5.8 \pm 0.19(n=39)$ & $<0.001$ & NA & NA & NA \\
\hline Emotion & $4.91 \pm 0.18(n=91)$ & $6.03 \pm 0.19(n=39)$ & $<0.001$ & NA & NA & NA \\
\hline Activity limitation & $3.91 \pm 0.15(n=91)$ & $4.57 \pm 0.19(n=39)$ & 0.012 & NA & NA & NA \\
\hline \multicolumn{7}{|l|}{ PACQLQ } \\
\hline Total & NA & NA & NA & $4.27 \pm 0.18(n=77)$ & $6.04 \pm 0.18(n=52)$ & $<0.001$ \\
\hline Total z-score & NA & NA & NA & $-0.46 \pm 0.09(n=77)$ & $0.66 \pm 0.12(n=52)$ & $<0.001$ \\
\hline \multicolumn{7}{|l|}{ Exacerbations } \\
\hline Total z-score & $-0.25 \pm 0.12(n=67)$ & $0.69 \pm 0.17(n=24)$ & $<0.001$ & NA & NA & NA \\
\hline Total $\leqslant 19$ & $50 / 67(74.6 \%)$ & $7 / 24(29.2 \%)$ & $<0.001$ & NA & NA & NA \\
\hline \multicolumn{7}{|l|}{ Childhood ACT } \\
\hline Total & $16.38 \pm 0.98(n=29)$ & $19.22 \pm 1.01(n=18)$ & 0.061 & $15.2 \pm 0.79(n=41)$ & $23 \pm 0.67(n=22)$ & $<0.001$ \\
\hline Total z-score & $-0.26 \pm 0.16(n=29)$ & $0.23 \pm 0.2(n=18)$ & 0.065 & $-0.47 \pm 0.13(n=41)$ & $1.01 \pm 0.16(n=22)$ & $<0.001$ \\
\hline Total $\leqslant 19$ & $19 / 29(65.5 \%)$ & $7 / 18(38.9 \%)$ & 0.078 & $32 / 41(78 \%)$ & $4 / 22(18.2 \%)$ & $<0.001$ \\
\hline \multicolumn{7}{|l|}{ Combined $\mathrm{ACT}^{\#}$} \\
\hline z-score & $-0.26 \pm 0.1(n=95)$ & $0.47 \pm 0.13(n=41)$ & $<0.001$ & $-0.47 \pm 0.13(n=41)$ & $1.01 \pm 0.16(n=22)$ & $<0.001$ \\
\hline
\end{tabular}

Data are presented as mean $\pm \mathrm{SE}$, median (interquartile range) or $\mathrm{n} / \mathrm{N}(\%)$, unless otherwise stated. $\mathrm{p}$-values were calculated using a Kruskall-Wallis test or Pearson's Chi-squared test. PAQLQ: Paediatric Asthma Quality of Life Questionnaire; PACQLQ: Paediatric Asthma Caregiver's Quality of Life Questionnaire; ACT: Asthma Control Test (used for participants >12 years; children aged 4-11 years completed the childhood ACT); NA: not applicable. \# : to allow the joint analysis of the ACT and childhood ACT, data were transformed to improve symmetry and then z-scores were calculated. 
TABLE 5 Factors associated with asthma burden as measured by quality of life

\begin{tabular}{|c|c|c|c|c|}
\hline & $\begin{array}{l}\text { Sample } \\
\text { size } n\end{array}$ & $\begin{array}{l}\text { Regression } \\
\text { coefficient }\end{array}$ & $\begin{array}{l}95 \% \text { confidence } \\
\text { interval }\end{array}$ & p-value \\
\hline FEV1 z-score ${ }^{\#}$ & 161 & 0.151 & $0.05-0.25$ & 0.002 \\
\hline FEV $1 / F V C$ & 162 & 1.113 & $-0.23-2.46$ & 0.104 \\
\hline Age of onset years & 247 & 0.028 & $-0.03-0.08$ & 0.311 \\
\hline Specific airway conductance $\mathrm{kPa} \cdot \mathrm{s}^{-1}$ & 78 & 0.132 & $-0.16-0.42$ & 0.366 \\
\hline Log exacerbations in previous year & 263 & -0.523 & $-0.67--0.38$ & $<0.001$ \\
\hline ACT combined z-score & 196 & 0.730 & $0.63-0.83$ & $<0.001$ \\
\hline $\mathrm{BMI} \mathbf{k g} \cdot \mathrm{m}^{-2}$ & 261 & -0.036 & $-0.06--0.01$ & 0.011 \\
\hline MARS total & 249 & -0.040 & $-0.09-0.01$ & 0.097 \\
\hline Hay fever diagnosed & 211 & -0.281 & $-0.57-0.01$ & 0.057 \\
\hline Eczema diagnosed & 225 & -0.116 & $-0.42-0.19$ & 0.452 \\
\hline Atopy & 225 & -0.070 & $-0.34-0.2$ & 0.612 \\
\hline Second-hand smoke & 257 & -0.022 & $-0.33-0.29$ & 0.890 \\
\hline White ethnicity & 263 & 0.346 & $0.04-0.66$ & 0.028 \\
\hline
\end{tabular}

Data represent linear regression analyses looking at the association between each factor and quality of life. Quality of life was assessed by the Paediatric Asthma Quality of Life Questionnaire (PAQLQ) or the Paediatric Asthma Caregiver's Quality of Life Questionnaire (PACQLQ). To allow the joint analysis of the PAQLQ and $P A C Q L Q$, data were transformed to improve symmetry and then z-scores were calculated. In order to make maximum use of the data, plethysmography (specific airway conductance), with 194 missing values, was excluded from joint modelling. Analysis was also performed for PAQLQ and PACQLQ separately (results in the online supplementary material). FEV1: forced expiratory volume in $1 \mathrm{~s}$; FVC: forced vital capacity; ACT: Asthma Control Test; BMI: body mass index; MARS: Medication Adherence Report Scale. " : predictive lung function equations from QUANJER et al. [19] were used to generate z-scores.

\section{Discussion}

This article presents the detailed clinical characteristics of 282 children in four paediatric cohorts, including preschool and school-age children with both severe and mild/moderate wheeze and asthma across Europe. Standard Operating Procedures and Good Clinical Practice criteria were used to ensure consistency and quality across sites, with data collected on an online platform and stored in a single online repository. The severe cohorts by definition had a significantly higher treatment burden than the mild/ moderate ones, despite which they remained poorly controlled with frequent severe exacerbations and low ACT scores. Children with severe disease, and their caregivers, had significantly lower QoL scores across all domains than the mild/moderate cohorts. Asthma control and airway obstruction were found to be significantly associated with QoL. Exposure to environmental tobacco smoke in the SW cohort was a striking finding and will be an important concomitant factor in future analyses. Otherwise, the severe and mild/moderate cohorts were very similar; this is in contrast to the adult severe and mild/moderate U-BIOPRED cohorts [11] and suggests that paediatricians should be cautious about extrapolating from adult studies. The vast majority of children were atopic. The rates of reported food allergy were high, although the rate of actual food allergy is expected to be much lower [24]. Most had a normal BMI, unlike the typical adult severe asthma phenotype. Also conspicuous was the morbidity in the mild/moderate paediatric groups; although they were clearly differentiated from the severe groups, a number are clearly sub-optimally treated. These data demonstrate that we succeeded in recruiting severe paediatric cohorts and provide a comprehensive view of the clinical burden of severe asthma or wheeze in childhood.

Children in the U-BIOPRED SA and SW cohorts have frequent symptoms and severe exacerbations that adversely impact on QoL and carry a high treatment burden; almost 17\% of the SA cohort was prescribed omalizumab and $24 \%$ prescribed maintenance oral corticosteroids. This is in keeping with a previous study, which reported a strong association between health-related QoL and ACT score in children with problematic severe asthma [25]. In our study, the impact on QoL was seen to be greatest for the SW cohort. A significant impact on QoL was also seen in the mild/moderate cohorts, highlighting the often overlooked influence of asthma on the lives of children and their families. Allergic sensitisation and other atopic diseases were a frequent finding across all cohorts, more so in school-aged children, adding further to the treatment burden. Lung function was not significantly different in the school-aged cohorts, possibly due to good treatment adherence, being between exacerbations and the fact that FEV1 is not a good discriminator of severity.

U-BIOPRED builds upon previous severe asthma cohort studies [26-29]. However, to our knowledge, this is the first study to recruit preschool wheeze cohorts on the basis of a consensus definition, which can be 

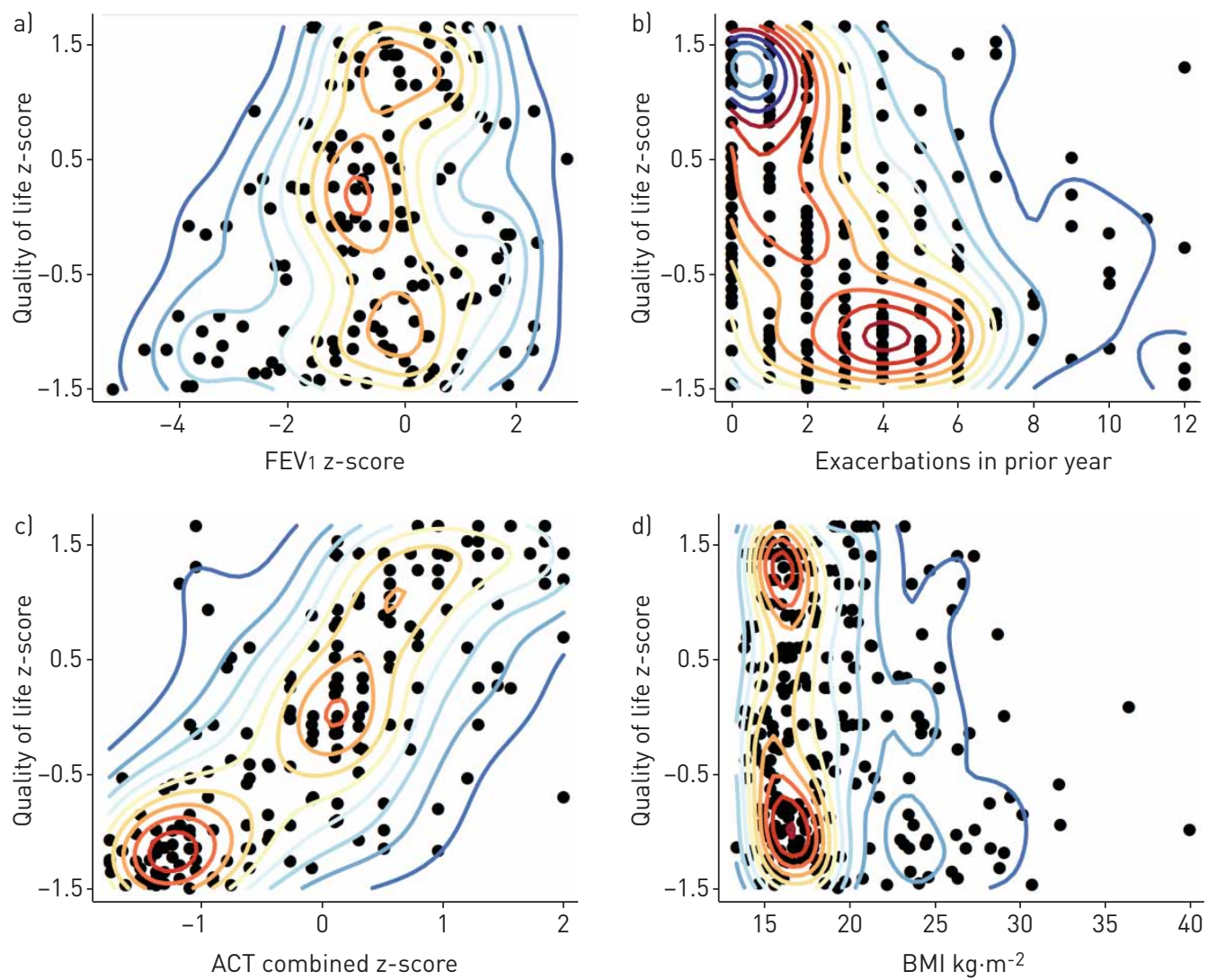

FIGURE 3 Factors associated with asthma burden as measured by quality of life. Figures represent scatter plots describing the relationship between each factor and the combined asthma-related quality of life z-score. The contour lines are coloured blue to red, to indicate increasing density of points in the graph to overcome the issue of overlying data points. The contour plots show c) a strong positive relationship between quality of life and asthma control (Asthma Control Test (ACT)) with a) a weaker positive relationship between quality of life and lung function (forced expiratory volume in $1 \mathrm{~s}\left(\mathrm{FEV}_{1}\right) \mathrm{z}$-score). Additionally there is b) a strong negative relationship between quality of life and exacerbation rate plus $\mathrm{d}$ ) a weaker negative relationship between quality of life and body mass index (BMI). The combined z-score merges the Paediatric Asthma Quality of Life Questionnaire (PAQLQ) and the Paediatric Asthma Caregiver's Quality of Life Questionnaire (PACQLQ). The density was modelled using two-dimensional kernel density estimation.

directly compared with parallel school-age and adult cohorts [13]. Most studies of preschool wheeze have been based on birth cohorts. A small number of studies have focused on severe preschool wheeze [9, 30] and they have provided valuable insights into the underlying pathophysiology and natural history of preschool wheeze. In common with the TENOR (The Epidemiology and Natural History of Asthma: Outcomes and Treatment Regimens) [27] and SARP (Severe Asthma Research Program) [26, 28] severe asthma cohorts, U-BIOPRED children with severe asthma were commonly atopic, had high healthcare utilisation and a high treatment burden. In the TENOR study there were far more boys than girls (63\% versus 37\%) in the severe cohort but, in common with SARP, we did not see these sex differences. Unlike SARP, children in the SA cohort did not have significantly higher FeNO levels than those in the MMA cohort; however, FeNO measurements were made off-line in SARP, making it difficult to make direct comparisons.

There are a number of limitations to this study. There were no healthy controls recruited to the paediatric cohorts; however, as the aim was to understand what makes asthma severe, the mild/moderate cohorts are the most appropriate comparator. The mild/moderate asthma group were all on prophylactic medication and participants were recruited from general paediatric and respiratory clinics so they are not completely representative of the children with mild/moderate asthma or wheeze seen in primary care. Also, as this is a multicentre pan-European study, it is likely that there were differences in patients recruited into each cohort between centres. Feasibility and safety considerations meant that assessments such as airway hyperresponsiveness were not included. Additionally, preschool children were unable to perform lung function, induced sputum and FeNO. We were not able to reach the target of 100 preschool severe wheeze children; many had not been under tertiary follow-up for $\geqslant 6$ months, did not reach the treatment threshold or did not meet the stringent inclusion criteria at screening due to the intermittent nature of 
their symptoms. There was no objective measure of adherence during the study; however, this was a pragmatic study of real-life severe asthma where clinics had tried to exclude adherence issues, and the high MARS scores suggest a good level of adherence.

Despite advances in recent years in our understanding and management of severe asthma, the data presented here highlight the ongoing unmet needs. Both severe asthma and severe wheeze are heterogeneous diseases. Single or even clustered biomarkers have had limited impact in predicting clinical course or therapeutic efficacy in children: for example, the SA cohort is not distinguishable from MMA by classical lung function and airway inflammatory phenotypes $[5,7,9]$. Classification of preschool wheeze phenotypes is at an even more basic level, limited to symptom pattern [31,32] and progression to asthma determined retrospectively. Analysis of samples from these cohorts will provide high-dimensional biological ("omics") data, which can be integrated with clinical characteristics to define multidimensional handprints of severe asthma. This approach has the potential to allow a step change in our understanding of asthma, identify more relevant prognostic and therapeutic targets and enable a personalised, phenotype-driven approach to management to address the unmet burden.

\section{Acknowledgements}

This paper is presented on behalf of the U-BIOPRED Study Group with input from the U-BIOPRED Patient Input Platform, Ethics Board and Safety Management Board.

The members of the U-BIOPRED Study Group are as follows: I.M. Adcock, Airways Disease Section, National Heart and Lung Institute, Imperial College London, London, UK; H. Ahmed, European Institute for Systems Biology and Medicine, University of Lyon, France; D. Allen, North West Severe Asthma Network; Pennine Acute Hospital NHS Trust; C. Auffray, European Institute for Systems Biology and Medicine, University of Lyon, France; P. Badorrek, Fraunhofer ITEM; S. Ballereau, European Institute for Systems Biology and Medicine, University of Lyon, France; F. Baribaud, Janssen R\&D, USA; M.K. Batuwitage, Imperial College, London, UK; A. Bedding, Roche Diagnostics GmbH, Mannheim, Germany; A.F. Behndig, Umeå University; E.H. Bel, Dept. Respiratory Medicine, Academic Medical Centre, University of Amsterdam, Amsterdam, The Netherlands; A. Berglind, Karolinska University Hospital and Karolinska Institutet; A. Berton, Boehringer Ingelheim Pharma GmbH \& Co. KG; J. Bigler, Amgen Inc; M.J. Boedigheimer, Amgen Inc; K. Bønnelykke, University of Copenhagen and Danish Pediatric Asthma Center, Gentofte Hospital, University of Copenhagen, Denmark; P. Brinkman, Academic Medical Centre, University of Amsterdam; E. Bucchioni, Chiesi Pharmacueticals SPA, Parma, Italy; D. Campagna, University of Catania; M. Caruso, Department of Clinical and Experimental Medicine Hospital University, University of Catania, Italy; C. Casaulta, University Children's Hospital Bern, Switzerland; A. Chaiboonchoe, European Institute for Systems Biology and Medicine, University of Lyon, France; P. Chanez, Département des Maladies Respiratoires, Laboratoire d'immunologie, Aix Marseille Université Marseille, France; C.H. Compton, Novartis Pharma, Basel, Switzerland; B. Dahlén, Lung/Allergy Clinic, Karolinska University Hospital Huddinge, Stockholm, Sweden; S.E. Dahlen, The Centre for Allergy Research, The Institute of Environmental Medicine, Karolinska Institutet, Stockholm, Sweden; T. Davison, Janssen R\&D, USA; B. De Meulder, European Institute for Systems Biology and Medicine, University of Lyon, France; I. Delin, Institute of Environmental Medicine, Karolinska Instituet, Stockholm, Sweden; P. Dennison, NIHR Southampton Respiratory Biomedical Research Unit and University of Southampton; R. Djukanovic, Southampton NIHR Respiratory Biomedical Research Unit, Southampton University Hospital. Southampton, UK; P. Dodson, AstraZeneca, Mölndal, Sweden; K. Dyson, CROMSOURCE, Stirling, UK; L. El Hadjam, European Institute for Systems Biology and Medicine, University of Lyon, France; V.J. Erpenbeek, Translational Medicine - Respiratory Profiling, Novartis Institutes for BioMedical Research, Basel, Switzerland; D. Erzen, Boehringer Ingelheim Pharma GmbH \& Co. KG; C. Faulenbach, Fraunhofer ITEM; K. Fichtner, Boehringer Ingelheim Pharma GmbH \& Co. KG; N. Fitch, BioSci Consulting, Belgium; M. Gahlemann, Boehringer Ingelheim (Schweiz) GmbH; G. Galffy, Semmelweis University, Budapest, Hungary; D. Garissi, Global Head Clinical Research Division, CROMSOURCE, Italy; T. Garret, BioSci Consulting, Belgium; T. Geiser, University Hospital Bern, Switzerland; J. Gent, Royal Brompton and Harefield NHS Foundation Trust; M. Gerhardsson de Verdier, AstraZeneca; Mölndal, Sweden; D. Gibeon, National Heart and Lung Institute, Imperial College, London, UK; E. Guillmant-Farry, Royal Brompton Hospital, London, UK; Y. Guo, Data Science Institute/Discovery Sciences Group, Hammersmith Hospital Campus, Imperial College London, UK; P.W. Hekking, Academic Medical Centre, University of Amsterdam; E. Henriksson, Karolinska Institutet; T. Higenbottam, Allergy Therapeutics, West Sussex, UK; U. Hoda, Imperial College; J.M. Hohlfeld, Fraunhofer ITEM; I. Horváth, Department of Pulmonology, Semmelweis University, Budapest, Hungary; P. Howarth, Southampton NIHR Respiratory Biomedical Research Unit, Southampton University Hospital. Southampton, UK; X. Hu, Amgen Inc; A. James, Karolinska Institutet; E. Jeyasingham, UK Clinical Operations, GSK, Stockley Park, UK; K. Johnson, Centre for respiratory medicine and allergy, Institute of Inflammation and repair, University Hospital of South Manchester, NHS Foundation Trust, Manchester, UK; N. Jullian, European Institute for Systems Biology and Medicine, University of Lyon, France; G. Kerry, Centre for respiratory medicine and allergy, Institute of Inflammation and repair, University Hospital of South Manchester, NHS Foundation Trust, Manchester, UK; M. Klüglich, Boehringer Ingelheim Pharma GmbH \& Co. KG; R. Knowles, Arachos Pharma, Stevenge, UK; A.J. Knox, Respiratory Research Unit, University of Nottingham, Nottingham, UK; J.R. Konradsen, Karolinska University Hospital and Karolinska Institutet; K. Kretsos, UCB, Slough, UK; L. Krueger, University Children's Hospital Bern, Switzerland; N. Krug, Fraunhofer Institute for Toxicology and Experimental Medicine Hannover, Germany; A-S. Lantz, Karolinska University Hospital and Karolinska Institutet; C. Larminie, GSK, London, UK; L.X. Larsson, AstraZeneca R\&D Molndal, Sweden; P. Latzin, University Children's Hospital Bern, Bern, Switzerland; N. Lazarinis, Lung/ Allergy Clinic, Karolinska University Hospital Huddinge, Stockholm, Sweden; D. Lefaudeux, European Institute for Systems Biology and Medicine, University of Lyon, France; N. Lemonnier, European Institute for Systems Biology and Medicine, University of Lyon, France; L.A. Lowe, Centre for respiratory medicine and allergy, Institute of Inflammation and repair, University Hospital of South Manchester, NHS Foundation Trust, Manchester, UK; R. Lutter, Academic Medical Centre, University of Amsterdam; A. Manta, Roche Diagnostics GmbH, Mannheim, Germany; J.G. Matthews, Respiratory and Allergy Diseases, Genentech, San Francisco, USA; A. Mazein, European Institute for Systems Biology and 
Medicine, University of Lyon, France; L. McEvoy, University Hospital, Department of Pulmonary Medicine, Bern, Switzerland; A. Menzies-Gow, Royal Brompton and Harefield NHS Foundation Trust; R.J.M. Middelveld, Karolinska Institutet; P. Montuschi, Faculty of Medicine, Catholic University of the Sacred Heart, Rome, Italy; N. Mores, Università Cattolica del Sacro Cuore; J. Musial, Dept. of Medicine, Jagiellonian University Medical College, Krakow, Poland; D. Myles, Respiratory Therapy Area Unit, GlaxoSmithKline, Stevenage, Hertfordshire, UK; K. Nething, Boehringer Ingelheim Pharma GmbH \& Co. KG; U. Nihlén, Department of Respiratory Medicine and Allergology, Skåne University Hospital, Lund, Sweden; AstraZeneca R\&D, Mölndal, Sweden; R. Niven, North West Severe Asthma Network, University Hospital South Manchester NHS Trust; B. Nordlund, Astrid Lindgren Children's Hospital, Karolinska University Hospital, Stockholm, Sweden; Department of Women's and Children's Health, Karolinska Institutet, Stockholm, Sweden; S. Nsubuga, Royal Brompton Hospital, London, UK; L. Pahus, Assistance publique des Hôpitaux de Marseille, Hôpital NORD, Clinique des bronches, allergies et sommeil, Marseille, France; J. Pellet, European Institute for Systems Biology and Medicine, University of Lyon, France; C. Pison, European Institute for Systems Biology and Medicine, University of Lyon, France; G. Praticò, CROMSOURCE, Verona, Italy; M. Puig Valls, CROMSOURCE, Barcelona, Spain; K. Riemann, Boehringer Ingelheim Pharma GmbH \& Co. KG; J.P. Rocha, Royal Brompton and Harefield NHS Foundation Trust; C. Rossios, Imperial College; T. Sandström, Dept. of Medicine, Respiratory and Allergy unit, University Hospital, Umeå, Sweden; G. Santini, Università Cattolica del Sacro Cuore; M. Saqi, European Institute for Systems Biology and Medicine, University of Lyon, France; S. Scott, North West Severe Asthma Network; Countess of Chester NHS Trust; N. Sehgal, North West Severe Asthma Network; Pennine Acute Hospital NHS Trust; W. Seibold, Boehringer Ingelheim Pharma GmbH \& Co.; R. Sigmund, Boehringer Ingelheim Pharma GmbH \& Co. KG; P. Söderman, Astrid Lindgren Children's Hospital, Karolinska University Hospital, Stockholm, Sweden; Department of Women's and Children's Health, Stockholm, Sweden; A. Sogbesan, Royal Brompton and Harefield NHS Foundation Trust; F. Spycher, University Hospital, Department of Pulmonary Medicine, Bern, Switzerland; S. Stephan, Centre for respiratory medicine and allergy, Institute of Inflammation and repair, University Hospital of South Manchester, NHS Foundation Trust, Manchester, UK; J. Stokholm, University of Copenhagen and Danish Pediatric Asthma Center, Gentofte Hospital, University of Copenhagen, Denmark; K. Strandberg, Karolinska University Hospital and Karolinska Institutet; M. Sunther, Centre for respiratory medicine and allergy, Institute of Inflammation and repair, University Hospital of South Manchester, NHS Foundation Trust, Manchester, UK; M. Szentkereszty, Semmelweis University, Budapest, Hungary; L. Tamasi, Semmelweis University, Budapest, Hungary; K. Tariq, NIHR Southampton Respiratory Biomedical Research Unit and University of Southampton; S. Valente, Università Cattolica del Sacro Cuore; C.M. van Drunen, Academic Medical Centre, University of Amsterdam; J. Van Eyll, UCB, Slough, UK; J. Vestbo, Respiratory Research Group, Manchester Academic Health Science Centre, University Hospital South Manchester NHS Foundation Trust, Manchester, UK; C. von Garnier, University Hospital Bern, Tiefenau Hospital Bern, Switzerland; A. Vyas, North West Severe Asthma Network; Lancashire Teaching Hospitals NHS Trust; A.H. Wagener, Academic Medical Center Amsterdam, Department of Respiratory Medicine, Amsterdam, The Netherlands; W. Yu, Amgen Inc; W. Zetterquist, Department of Woman and Child Health, Karolinska Institutet, Stockholm, Sweden; A.H. Zwinderman, Academic Medical Centre, University of Amsterdam.

The members of the U-BIOPRED Paediatric Study Group are as follows. Ann Berglind, Jon R. Konradsen and Päivi Söderman: Karolinska University Hospital \& Karolinska Institutet, Sweden; Klaus Bønnelykke, Nadja H. Vissing and Jakob Stokholm: University of Copenhagen \& Danish Pediatric Asthma Center, Gentofte Hospital, University of Copenhagen, Denmark; Gina Kerry, Lesley Lowe, Katy Johnson, Simon Stephan and Meera Sunthar: University of Manchester, UK; Emily Guillmant-Farry and Sheila Nsubuga: Royal Brompton and Harefield NHS Foundation Trust, UK.

Investigators and contributors: Nora Adriaens, Academic Medical Centre, University of Amsterdam, Amsterdam, The Netherlands; Antonios Aliprantis, Merck Research Laboratories, Boston, USA; Kjell Alving, Dept Women's andChildren's Health, Uppsala University, Uppsala, Sweden; Per Bakke, Department of Clinical Science, University of Bergen, Bergen, Norway; David Balgoma, Centre for Allergy Research, Karolinska Institutet, Stockholm, Sweden; Clair Barber, NIHR Southampton Respiratory Biomedical Research Unit and Clinical and Experimental Sciences, Southampton, UK; Frédéric Baribaud, Janssen R\&D, USA; Stewart Bates, Respiratory Therapeutic Unit, GSK, London, UK; An Bautmans, MSD, Brussels, Belgium; Jorge Beleta, Almirall S.A., Barcelona, Spain; Grazyna Bochenek, II Department of Internal Medicine, Jagiellonian University Medical College, Krakow, Poland; Joost Brandsma, University of Southampton, Southampton, UK; Armin Braun, Fraunhofer Institute for Toxicology and Experimental Medicine, Hannover, Germany; Dominic Burg, Centre for Proteomic Research, Institute for Life Sciences, University of Southampton, Southampton, UK; Leon Carayannopoulos, previously at: MSD, USA; João Pedro Carvalho da Purificação Rocha, Royal Brompton and Harefield NHS Foundation Trust, London, UK; Romanas Chaleckis, Centre of Allergy Research, Karolinska Institutet, Stockholm, Sweden; Courtney Coleman, Asthma UK, London, UK; Arnaldo D'Amico, University of Rome 'Tor Vergata', Rome Italy; Jorge De Alba, Almirall S.A., Barcelona, Spain; Inge De Lepeleire, MSD, Brussels, Belgium; Tamara Dekker, Academic Medical Centre, University of Amsterdam, Amsterdam, The Netherlands; Annemiek Dijkhuis, Academic Medical Centre, University of Amsterdam, Amsterdam, The Netherlands; Aleksandra Draper, BioSci Consulting, Maasmechelen, Belgium; Jessica Edwards, Asthma UK, London, UK; Rosalia Emma, Department of Clinical and Experimental Medicine, University of Catania, Catania, Italy; Magnus Ericsson, Karolinska University Hospital, Stockholm, Sweden; Breda Flood, European Federation of Allergy and Airways Diseases Patient's Associations, Brussels, Belgium; Urs Frey, University Children's Hospital, Basel, Switzerland; Hector Gallart, Centre for Allergy Research, Karolinska Institutet, Stockholm, Sweden; Cristina Gomez, Centre for Allergy Research, Karolinska Institutet, Stockholm, Sweden; Kerry Gove, NIHR Southampton Respiratory Biomedical Research Unit and Clinical and Experimental Sciences, Southampton, UK; Neil Gozzard, UCB, Slough, UK; John Haughney, International Primary Care Respiratory Group, Aberdeen, Scotland; Lorraine Hewitt, NIHR Southampton Respiratory Biomedical Research Unit, Southampton, UK; Tim Higgenbottam, Allergy Therapeutics, West Sussex, UK; Cecile Holweg, Respiratory and Allergy Diseases, Genentech, San Francisco, USA; Richard Hu, Amgen Inc. Thousand Oaks, USA; Sile Hu, National Heart and Lung Institute, Imperial College, London, UK; Juliette Kamphuis, Longfonds, Amersfoort, The Netherlands; Erika J. Kennington, Asthma UK, London, UK; Dyson Kerry, CromSource, Stirling, UK; Hugo Knobel, Philips Research Laboratories, Eindhoven, The Netherlands; Johan Kolmert, Centre for Allergy Research, Karolinska Institutet, Stockholm, Sweden; Maxim Kots, Chiesi Pharmaceuticals, SPA, Parma, Italy; Scott Kuo, National Heart and Lung Institute, Imperial College, London, UK; Maciej Kupczyk, Centre for Allergy Research, Karolinska Institutet, Stockholm, Sweden; Bart Lambrecht, University of Gent, Gent, Belgium; Saeeda Lone-Latif, Academic Medical Centre, University of Amsterdam, Amsterdam, The Netherlands; Matthew J. Loza, Janssen R\&D, USA; Lisa Marouzet, NIHR Southampton 
Respiratory Biomedical Research Unit, Southampton, UK; Jane Martin, NIHR Southampton Respiratory Biomedical Research Unit, Southampton, UK; Sarah Masefield, European Lung Foundation, Sheffield, UK; Caroline Mathon, Centre of Allergy Research, Karolinska Institutet, Stockholm, Sweden; Sally Meah, National Heart and Lung Institute, Imperial College, London, UK; Andrea Meiser, Data Science Institute, Imperial College, London, UK; Leanne Metcalf, previously at: Asthma UK, London, UK; Maria Mikus, Science for Life Laboratory and The Royal Institute of Technology, Stockholm, Sweden; Montse Miralpeix, Almirall, Barcelona, Spain; Philip Monk, Synairgen Research Ltd, Southampton, UK; Shama Naz, Centre for Allergy Research, Karolinska Institutet, Stockholm, Sweden; Ben Nicholas, University of Southampton, Southampton, UK; Peter Nilsson, Science for Life Laboratory and The Royal Institute of Technology, Stockholm, Sweden; Jörgen Östling, AstraZeneca, Mölndal, Sweden; Antonio Pacino, Lega Italiano Anti Fumo, Catania, Italy; Susanna Palkonen, European Federation of Allergy and Airways Diseases Patient's Associations, Brussels, Belgium; Stelios Pavlidis, National Heart and Lung Institute, Imperial College, London, UK; Giorgio Pennazza, University of Rome 'Tor Vergata', Rome Italy; Anne Petrén, Centre for Allergy Research, Karolinska Institutet, Stockholm, Sweden; Sandy Pink, NIHR Southampton Respiratory Biomedical Research Unit, Southampton, UK; Anthony Postle, University of Southampton, UK; Pippa Powell, European Lung Foundation, Sheffield, UK; Malayka Rahman-Amin, previously at: Asthma UK, London, UK; Navin Rao, Janssen R\&D, USA; Lara Ravanetti, Academic Medical Centre, University of Amsterdam, Amsterdam, The Netherlands; Emma Ray, NIHR Southampton Respiratory Biomedical Research Unit, Southampton, UK; Stacey Reinke, Centre for Allergy Research, Karolinska Institutet, Stockholm, Sweden; Leanne Reynolds, previously at: Asthma UK, London, UK; John Riley, Respiratory Therapeutic Unit, GSK, London, UK; Martine Robberechts, MSD, Brussels, Belgium; Amanda Roberts, Asthma UK, London, UK; Kirsty Russell, National Heart and Lung Institute, Imperial College, London, UK; Michael Rutgers, Longfonds, Amersfoort, The Netherlands; Marco Santoninco, University of Rome 'Tor Vergata', Rome Italy; Corinna Schoelch, Boehringer Ingelheim Pharma GmbH \& Co. KG, Biberach, Germany; James P.R. Schofield, Centre for Proteomic Research, Institute for Life Sciences, University of Southampton, Southampton, UK; Marcus Sjödin, Centre for Allergy Research, Karolinska Institutet, Stockholm, Sweden; Paul J. Skipp, Centre for Proteomic Research, Institute for Life Sciences, University of Southampton, Southampton, UK; Barbara Smids, Academic Medical Centre, University of Amsterdam, Amsterdam, The Netherlands; Caroline Smith, NIHR Southampton Respiratory Biomedical Research Unit, Southampton, UK; Jessica Smith, Asthma UK, London, UK; Katherine M. Smith, University of Nottingham, UK; Adesimbo Sogbesan, Royal Brompton and Harefield NHS Foundation Trust, London, UK; Doroteya Staykova, University of Southampton, Southampton, UK; Kai Sun, Data Science Institute, Imperial College, London, UK; David Supple, Asthma UK, London, UK; John-Olof Thörngren, Karolinska University Hospital, Stockholm, Sweden; Bob Thornton, MSD, USA; Jonathan Thorsen, COPSAC, Copenhagen Prospective Studies on Asthma in Childhood, Herlev and Gentofte Hospital, University of Copenhagen, Copenhagen, Denmark; Marianne van de Pol, Academic Medical Centre, University of Amsterdam, Amsterdam ,The Netherlands; Marleen van Geest, AstraZeneca, Mölndal, Sweden; Jenny Versnel, previously at: Asthma UK, London, UK; Anton Vink, Philips Research Laboratories, Eindhoven, The Netherlands; Frans Wald, Boehringer Ingelheim Pharma GmbH \& Co. KG, Biberach, Germany; Samantha Walker, Asthma UK, London, UK; Jonathan Ward, Histochemistry Research Unit, Faculty of Medicine, University of Southampton, Southampton, UK; Zsoka Weiszhart, Semmelweis University, Budapest, Hungary; Kristiane Wetzel, Boehringer Ingelheim Pharma GmbH, Biberach, Germany; Craig E. Wheelock, Centre for Allergy Research, Karolinska Institutet, Stockholm, Sweden; Coen Wiegman, National Heart and Lung Institute, Imperial College, London, UK; Siân Williams, International Primary Care Respiratory Group, Aberdeen, Scotland; Susan J. Wilson, Histochemistry Research Unit, Faculty of Medicine, University of Southampton, Southampton, UK; Ashley Woodcock, Centre for Respiratory Medicine and Allergy, Institute of Inflammation and Repair, University of Manchester and University Hospital of South Manchester, Manchester Academic Health Sciences Centre, Manchester, UK; Xian Yang, Data Science Institute, Imperial College, London, UK; Elizabeth Yeyasingham, UK Clinical Operations, GSK, Stockley Park, UK.

The authors would like to acknowledge Elizabeth Juniper (McMaster University, Hamilton, ON, Canada) (PAQLQ and PACQLQ) and Robert Horne (University College London, London, UK) (MARS) for the use of their questionnaires. They would also like to acknowledge help in data and knowledge management from the eTRIKS project, which is funded by the Innovative Medicines Initiative. Additionally, the authors would like to acknowledge the support of the University Hospital NHS Foundation Trust and the NIHR-Wellcome Trust Clinical Research Facility, Southampton, UK.

\section{References}

1 ChungKF, Wenzel SE, Brozek JL, et al. International ERS/ATS guidelines on definition, evaluation and treatment of severe asthma. Eur Respir J 2014; 43: 343-373.

2 Wenzel SE. Asthma: defining of the persistent adult phenotypes. Lancet 2006; 368: 804-813.

3 Fitzpatrick AM, Teague WG, Meyers DA, et al. Heterogeneity of severe asthma in childhood: confirmation by cluster analysis of children in the National Institutes of Health/National Heart, Lung, and Blood Institute Severe Asthma Research Program. J Allergy Clin Immunol 2011; 127: 382-389.

4 Just J, Gouvis-Echraghi R, Rouve S, et al. Two novel, severe asthma phenotypes identified during childhood using a clustering approach. Eur Respir J 2012; 40: 55-60.

5 Bossley CJ, Fleming L, Gupta A, et al. Pediatric severe asthma is characterized by eosinophilia and remodeling without $\mathrm{T}_{\mathrm{H}} 2$ cytokines. J Allergy Clin Immunol 2012; 129: 974-982.

6 Hastie AT, Moore WC, Meyers DA, et al. Analyses of asthma severity phenotypes and inflammatory proteins in subjects stratified by sputum granulocytes. J Allergy Clin Immunol 2010; 125: 1028-1036.

7 Fleming L, Tsartsali L, Wilson N, et al. Sputum inflammatory phenotypes are not stable in children with asthma. Thorax 2012; 67: 675-681.

8 Saglani S, Payne DN, Zhu J, et al. Early detection of airway wall remodeling and eosinophilic inflammation in preschool wheezers. Am J Respir Crit Care Med 2007; 176: 858-864.

9 O'Reilly R, Ullmann N, Irving S, et al. Increased airway smooth muscle in preschool wheezers who have asthma at school age. J Allergy Clin Immunol 2013; 131: 1024-1032.

10 Auffray C, Adcock IM, Chung KF, et al. An integrative systems biology approach to understanding pulmonary diseases. Chest 2010; 137: 1410-1416.

11 Shaw DE, Sousa AR, Fowler SJ, et al. Clinical and inflammatory characteristics of the European U-BIOPRED adult severe asthma cohort. Eur Respir J 2015; 46: 1308-1321. 
12 Wheelock CE, Goss VM, Balgoma D, et al. Application of 'omics technologies to biomarker discovery in inflammatory lung diseases. Eur Respir J 2013; 42: 802-825.

13 Bel EH, Sousa A, Fleming L, et al. Diagnosis and definition of severe refractory asthma: an international consensus statement from the Innovative Medicine Initiative (IMI). Thorax 2011; 66: 910-917.

14 Nathan RA, Sorkness CA, Kosinski M, et al. Development of the Asthma Control Test: a survey for assessing asthma control. J Allergy Clin Immunol 2004; 113: 59-65.

15 Liu AH, Zeiger R, Sorkness C, et al. Development and cross-sectional validation of the Childhood Asthma Control Test. J Allergy Clin Immunol 2007; 119: 817-825.

16 Juniper EF, Guyatt GH, Feeny DH, et al. Measuring quality of life in children with asthma. Qual Life Res 1996; 5: 35-46.

17 Juniper EF, Guyatt GH, Feeny DH, et al. Measuring quality of life in the parents of children with asthma. Qual Life Res 1996; 5: 27-34.

18 Cohen JL, Mann DM, Wisnivesky JP, et al. Assessing the validity of self-reported medication adherence among inner-city asthmatic adults: the Medication Adherence Report Scale for Asthma. Ann Allergy Asthma Immunol 2009; 103: 325-331.

19 Quanjer PH, Stanojevic S, Cole TJ, et al. Multi-ethnic reference values for spirometry for the 3-95-yr age range: the global lung function 2012 equations. Eur Respir J 2012; 40: 1324-1343.

20 Szalma S, Koka V, Khasanova T, et al. Effective knowledge management in translational medicine. J Transl Med 2010; 8: 68 .

21 Cohen J. Statistical Power Analysis for the Behavioral Sciences. 2nd Edn. Hillsdale, Lawrence Erlbaum Associates, 1988.

221990 British growth data. Available from www.healthforallchildren.com/?product=lmsgrowth Date last accessed: April 26, 2015

23 Venables WN, Ripley BD. Modern Applied Statistics with S. 4th Edn. New York, Springer, 2002.

24 Nwaru BI, Hickstein L, Panesar SS, et al. The epidemiology of food allergy in Europe: a systematic review and meta-analysis. Allergy 2014; 69: 62-75.

25 Nordlund B, Konradsen JR, Pedroletti C, et al. The clinical benefit of evaluating health-related quality-of-life in children with problematic severe asthma. Acta Paediatr 2011; 100: 1454-1460.

26 Fitzpatrick AM, Gaston BM, Erzurum SC, et al. Features of severe asthma in school-age children: atopy and increased exhaled nitric oxide. J Allergy Clin Immunol 2006; 118: 1218-1225.

27 Chipps BE, Szefler SJ, Simons FE, et al. Demographic and clinical characteristics of children and adolescents with severe or difficult-to-treat asthma. J Allergy Clin Immunol 2007; 119: 1156-1163.

28 Moore WC, Bleecker ER, Curran-Everett D, et al. Characterization of the severe asthma phenotype by the National Heart, Lung, and Blood Institute's Severe Asthma Research Program. J Allergy Clin Immunol 2007; 119: 405-413.

29 Konradsen JR, Nordlund B, Lidegran M, et al. Problematic severe asthma: a proposed approach to identifying children who are severely resistant to therapy. Pediatr Allergy Immunol 2011; 22: 9-18.

30 Saglani S, Nicholson AG, Scallan M, et al. Investigation of young children with severe recurrent wheeze: any clinical benefit? Eur Respir J 2006; 27: 29-35.

31 Brand PL, Baraldi E, Bisgaard H, et al. Definition, assessment and treatment of wheezing disorders in preschool children: an evidence-based approach. Eur Respir J 2008; 32: 1096-1110.

32 Brand PL, Caudri D, Eber E, et al. Classification and pharmacological treatment of preschool wheezing: changes since 2008. Eur Respir J 2014; 43: 1172-1177. 